\title{
SLICES, RNP, STRONG REGULARITY, AND MARTINGALES
}

\author{
Maria Girardi and J.J. UhL, JR.
}

\begin{abstract}
The usual proof that dentability implies the Radon-Nikodým property involves a clever but rather baroque exhaustion argument. This note presents a very short and simple proof of this implication. The techniques in this new proof are then generalised to derive some direct proofs of recent results concerning strongly regular operators on $L_{1}$.
\end{abstract}

The usual proof that dentability implies the Radon-Nikodým property (RNP) involves a clever but rather baroque exhaustion argument [2, pp. 136-138]. The main purpose of this note is to present a very short and simple proof of this implication. We then generalise the techniques in this new proof to derive some direct proofs of recent results of Ghoussoub, Godefroy, Maurey, and Schachermayer on bounded linear operators on $L_{1}(\mu)$.

The idea behind our approach is to study the behavior of such an operator on subsets of the form

$$
\Delta_{A} \equiv\left\{\frac{\chi_{E}}{\mu(E)} \in L_{1}(\mu): E \subset A \text { and } \mu(E)>0\right\}
$$

(which we call $A$-segments). Compare this approach with the usual approach [3] that considers subsets of the form

$$
\mathcal{F}_{A} \equiv\left\{f \in L_{1}(\mu): f \geqslant 0 \text { a.e. } \quad \text { and }\|f\|_{L_{1}}=\left\|f \chi_{A}\right\|_{L_{1}}=1\right\}=\overline{c o} \Delta_{A} \text {. }
$$

Our approach not only provides enough information about the operator but also often leads to simpler proofs. Our new proofs involving $A$-segments stem from the simple, yet until now unnoticed, lemma in the first section.

Throughout this note, $\mathfrak{X}$ is a Banach space, $B(\mathfrak{X})$ is the closed unit ball of $\mathfrak{X}$, and $(\Omega, \Sigma, \mu)$ is a finite measure space. The subset of $\Sigma$ consisting of those sets of positive $\mu$-measure is denoted by $\Sigma^{+}$. All notations and terminology, not otherwise explained, are as in [2].

Received 21st June 1989

Copyright Clearance Centre, Inc. Serial-fee code: 0004-9729/90 SA2.00+0.00. 


\section{RNP AND DENTABILITY}

Recall that $\mathfrak{X}$ has the RNP precisely if all bounded linear operators from $L_{1}(\mu)$ into $\mathfrak{X}$ are representable. To test the representability of such an operator $T$ it is sufficient [2, Lemma V.3.6] to find, for $\varepsilon>0$ and $A$ in $\Sigma^{+}$, a subset $B$ of $A$ such that $B \in \Sigma^{+}$ and

$$
\operatorname{diam} T \Delta_{B} \equiv \operatorname{diam}\left\{\frac{T \chi_{E}}{\mu(E)}: E \subset B \text { and } E \in \Sigma^{+}\right\}<\varepsilon .
$$

The usual proof that dentability implies the RNP uses a rather involved argument to find such a subset $B$ of $A$ and the following definition of dentability.

Definition: A subset $D$ of $\mathfrak{X}$ is dentable if for each $\varepsilon>0$ there is an $x$ in $D$ such that

$$
x \notin \overline{\mathrm{co}}\left(D \backslash B_{e}(x)\right)
$$

Here we present a short proof that dentability implies the RNP by finding such a subset $B$ of $A$ using the slice definition of dentability. Recall that a slice $S$ of a subset $D$ of $\mathfrak{X}$ is any non-empty set of the form

$$
S=\left\{x \in D: x^{*}(x)>\alpha\right\}
$$

where $x^{*}$ in $\mathfrak{X}^{*}$ and the real number $\alpha$ are fixed. The Hahn-Banach theorem guarantees that a bounded subset $D$ of $\mathfrak{X}$ is dentable precisely if it has slices of arbitrarily small diameter.

We now present this note's main lemma. While simple, it is very useful.

LEмmA . Let $T$ be a bounded linear operator from $L_{1}(\mu)$ into $\mathfrak{X}$. If $S$ is a slice of $T \Delta_{A}$ for some $A$ in $\Sigma^{+}$, then there is a subset $B$ of $A$ such that $B \in \Sigma^{+}$and $T \Delta_{B} \subset S$.

Proof: Let $S$ be a slice of $T \Delta_{A}$ for some $A$ in $\Sigma^{+}$. Accordingly, there is an $x^{*}$ in $X^{*}$ and a real number $\alpha$ such that

$$
S=\left\{\frac{T \chi_{E}}{\mu(E)}: x^{*}\left(\frac{T \chi_{E}}{\mu(E)}\right)>\alpha, E \subset A, E \in \Sigma^{+}\right\}
$$

Since $x^{*} T \chi_{(.)}: \Sigma \rightarrow R$ is a $\mu$-continuous measure, there is a real-valued function $g$ in $L_{1}(\mu)$ such that $x^{*} T \chi E=\int_{E} g d \mu$ for each $E$ in $\Sigma$. Set $B=[g>\alpha] \cap A$. Note that $B \in \Sigma^{+}$because $S$ is not empty.

We now give a very short and simple proof of a twenty-year-old theorem of Rieffel [2, pp. 136-138]. 
THEOREM. If every bounded subset of $\mathfrak{X}$ is dentable, then every bounded linear operator from $L_{1}$ into $\mathfrak{X}$ is representable and so $\mathfrak{X}$ has the RNP.

PROOF: Let all bounded subsets of $\mathfrak{X}$ be dentable. Let $T$ be a bounded linear operator from $L_{1}$ into $\mathfrak{X}$. Fix $\varepsilon>0$ and $A$ in $\Sigma^{+}$. Since $T \Delta_{A}$ is bounded, there is a slice $S$ of $T \Delta_{A}$ with diam $S<\varepsilon$. By the lemma, there is a subset $B$ of $A$ such that $B \in \Sigma^{+}$ and $T \Delta_{B} \subset S$, which clearly implies that $\operatorname{diam} T \Delta_{B}<\varepsilon$.

Possibly one reason this proof is so short is that, unlike the usual proofs, it makes use of the scalar version of the Radon-Nikodým theorem (see the proof of the lemma).

The ideas in our proof lend themselves to generalisations. We explore some of these generalisations and their applications in the next section.

\section{Strong Regularity}

Fix a bounded linear operator $T$ from $L_{1}(\mu)$ into $\mathfrak{X}$. Recall [3] that $T$ is strongly regular if for each bounded subset $D$ of $L_{1}(\mu)$ and $\varepsilon>0$ there are finitely many slices $S_{1}, \ldots, S_{n}$ of $D$ such that

$$
\operatorname{diam}\left[T\left(\sum_{i=1}^{n} \alpha_{i} S_{i}\right)\right]<\varepsilon,
$$

for a suitable choice of positive real numbers $\alpha_{1}, \ldots, \alpha_{n}$ whose sum is one.

In this section, using the following variation of the lemma in section 1 , we present several new, simple, and direct proofs of theorems from [3] about strongly regular operators.

Lemma. If $S$ is a slice of $\Delta_{A}$ for some $A$ in $\Sigma^{+}$, then there is a subset $B$ of $A$ such that $B \in \Sigma^{+}$and $\Delta_{B} \subset S$.

PRoof: Let $S$ be a slice of $\Delta_{A}$ for some $A$ in $\Sigma^{+}$. Accordingly, there are a real-valued function $g$ in $L_{\infty}(\mu)$ and a real number $\alpha$ such that

$$
S=\left\{\frac{\chi_{E}}{\mu(E)}: \frac{\int_{E} g d \mu}{\mu(E)}>\alpha, E \subset A, E \in \Sigma^{+}\right\}
$$

Set $B=[g>\alpha] \cap A$.

Remarks. (i) This lemma implies the lemma of section 1 for if one is given a slice $S$ of $T \Delta_{A}$, then one can find a slice $\tilde{S}$ of $\Delta_{A}$ such that $T \tilde{S}=S$.

(ii) This lemma is to $\Delta_{A}$ as $\left[3\right.$, Lemma IV.1] is to $\mathcal{F}_{A}$.

Recall that the subset $T^{*}\left(B\left(\mathfrak{X}^{*}\right)\right)$ of $L_{\infty}(\mu)$ is a set of small oscillation with respect to $\mu$ if for each $\varepsilon>0$ there is a finite measurable partition $\pi$ of $\Omega$ such that

$$
\left.\sup _{z^{*} \in B\left(X^{*}\right)} \sum_{A \in \pi} \mu(A) \operatorname{osc} T^{*} x^{*}\right|_{A}<\varepsilon .
$$


Using the lemma, we now give a direct proof of the following theorem [3].

TheoreM. If $T$ is strongly regular, then $T^{*}\left(B\left(\mathfrak{X}^{*}\right)\right)$ is a set of small oscillation with respect to $\mu$.

PROOF: Let $T$ be a strongly regular operator from $L_{1}(\mu)$ into $\mathfrak{X}$. Fix $\varepsilon>0$. Consider $A$ in $\Sigma^{+}$. By strong regularity there are slices $S_{1}, \ldots, S_{n}$ of the $\Delta_{A}$ and real numbers $\alpha_{1}, \ldots, \alpha_{n}$ whose sum is one such that

$$
\operatorname{diam}\left[T\left(\sum_{i=1}^{n} \alpha_{i} S_{i}\right)\right]<\varepsilon
$$

Use the lemma of this section to find subsets $B_{1}, \ldots, B_{n}$ of $A$ such that $B_{i}$ is in $\Sigma^{+}$ and $\Delta_{B_{i}} \subset S_{i}$ for each $i$. We now have that

$$
\operatorname{diam}\left[T\left(\sum_{i=1}^{n} \alpha_{i} \Delta_{B_{i}}\right)\right]<\varepsilon
$$

Set $B=\cup_{i=1}^{n} B_{i}$. A moment's reflection shows that without loss of generality the sets $B_{1}, \ldots, B_{n}$ are disjoint. A second moment of reflection shows that we may chop back the $B_{i}$ 's so that $\alpha_{i} \mu(B)=\mu\left(B_{i}\right)$ for each $i$. From this it follows that

$$
\operatorname{diam}\left[T\left(\sum_{i=1}^{n} \mu\left(B_{i}\right) \Delta_{B_{i}}\right)\right]<\varepsilon \mu(B)
$$

Since we can apply the same argument with $A$ replaced by $\Omega \backslash B$, we can perform a standard exhaustion procedure to produce a positive measurable partition $\pi=$ $\left\{B_{1}, \ldots, B_{n}\right\}$ of $\Omega$ such that

$$
\operatorname{diam}\left[T\left(\sum_{i=1}^{n} \mu\left(B_{i}\right) \Delta_{B_{i}}\right)\right]<\varepsilon \mu(\Omega)
$$

But since the $B_{i}$ 's are disjoint,

$$
\operatorname{diam}\left[T\left(\sum_{i=1}^{n} \mu\left(B_{i}\right) \Delta_{B_{i}}\right)\right]=\left.\sup _{x^{*} \in B\left(X^{*}\right)} \sum_{i=1}^{n} \mu\left(B_{i}\right) \operatorname{osc} T^{*} x^{*}\right|_{B_{i}}
$$

Thus $T^{*}\left(B\left(\mathfrak{X}^{*}\right)\right)$ is indeed a set of small oscillation.

The lemma also provides a simple proof of the following well-known fact. 
Theorem. If $T$ is strongly regular, then $T$ is Dunford-Pettis.

PROOF: Let $T$ be a strongly regular operator from $L_{1}(\mu)$ into $\mathfrak{X}$. Define the usual "difference quotient" martingale

$$
f_{\pi}=\sum_{E \in \pi} \frac{T \chi_{E}}{\mu(E)} \chi_{E}
$$

where $\pi$ ranges over all finite positive measurable partitions of $\Omega$. Fix $\varepsilon>0$. As in the proof of the previous theorem, find a positive measurable partition $\pi=\left\{B_{1}, \ldots, B_{n}\right\}$ of $\Omega$ such that

$$
\operatorname{diam}\left[T\left(\sum_{i=1}^{n} \mu\left(B_{i}\right) \Delta_{B_{i}}\right)\right]<\varepsilon \mu(\Omega) .
$$

If the partition $\tau$ refines $\pi$ and $x^{*} \in B\left(X^{*}\right)$, then since the $B_{i}$ 's are disjoint

$$
\begin{aligned}
\int_{\Omega}\left|x^{*}\left(f_{\tau}-f_{\pi}\right)\right| d \mu & \leqslant \sum_{i=1}^{n} \mu\left(B_{i}\right) \operatorname{diam} x^{*} T \Delta_{B_{i}} \\
& =\operatorname{diam}\left[\sum_{i=1}^{n} \mu\left(B_{i}\right) x^{*} T \Delta_{B_{i}}\right] \\
& \leqslant \operatorname{diam}\left[\sum_{i=1}^{n} \mu\left(B_{i}\right) T \Delta_{B_{i}}\right] \\
& <\varepsilon \mu(\Omega) .
\end{aligned}
$$

So the martingale $\left\{f_{\pi}\right\}$ is Pettis-Cauchy; hence, $T$ is Dunford-Pettis [1].

\section{REFERENCES}

[1] J. Bourgain, 'Dunford-Pettis operators on $L_{1}$ and the Radon-Nikodým property', Israel J. Math. 37 (1980), 34-47.

[2] J. Diestel and J. J. Uhl, Jr., Vector Measures, Math. Surveys, no. 15 (American Mathethematical Society, Providence, R.I., 1977).

[3] N. Ghoussoub, G. Godefroy, B. Maurey, and W. Schachermayer, 'Some topological and geometrical structures in Banach spaces', in Mem. American Mathematical Society, 70, no. 378 (Amer. Math. Soc., Providence, R.I., 1987). 